\title{
Transepithelial Transport of Curcumin in Caco-2 Cells Is significantly Enhanced by Micellar Solubilisation
}

\author{
Jan Frank $^{1}$ - Christina Schiborr ${ }^{1}$ - Alexa Kocher ${ }^{1}$. Jürgen Meins ${ }^{2}$ - Dariush Behnam ${ }^{3}$. \\ Manfred Schubert-Zsilavecz ${ }^{2,4}$ • Mona Abdel-Tawab ${ }^{2}$
}

Published online: 29 November 2016

(C) The Author(s) 2016. This article is published with open access at Springerlink.com

\begin{abstract}
Curcumin, the active constituent of Curcuma longa L. (family Zingiberaceae), has gained increasing interest because of its anti-cancer, anti-inflammatory, anti-diabetic, and anti-rheumatic properties associated with good tolerability and safety up to very high doses of $12 \mathrm{~g}$. Nanoscaled micellar formulations on the base of Tween 80 represent a promising strategy to overcome its low oral bioavailability. We therefore aimed to investigate the uptake and transepithelial transport of native curcumin (CUR) vs. a nanoscaled micellar formulation (Sol-CUR) in a Caco-2 cell model. Sol-CUR afforded a higher

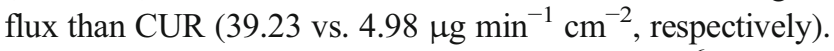
This resulted in a higher $\mathrm{P}_{\text {app }}$ value of $2.11 \times 10^{-6} \mathrm{~cm} / \mathrm{s}$ for Sol-CUR compared to a $\mathrm{P}_{\text {app }}$ value of $0.56 \times 10^{-6} \mathrm{~cm} / \mathrm{s}$ for CUR. Accordingly a nearly 9.5 fold higher amount of curcumin was detected on the basolateral side at the end of the transport experiments after $180 \mathrm{~min}$ with Sol-CUR compared to CUR. The determined 3.8-fold improvement in the permeability of curcumin is in agreement with an up to 185fold increase in the AUC of curcumin observed in humans following the oral administration of the nanoscaled micellar formulation compared to native curcumin. The present study demonstrates that the enhanced oral bioavailability of micellar curcumin formulations is likely a result of enhanced
\end{abstract}

Mona Abdel-Tawab

m.tawab@zentrallabor.com

1 Institute of Biological Chemistry and Nutrition, University of Hohenheim, D-70599 Stuttgart, Germany

2 Central Laboratory of German Pharmacists, Carl-Mannich-Str. 20, D-65760 Eschborn, Germany

3 AQUANOVA AG, D-64295 Darmstadt, Germany

4 Institute of Pharmaceutical Chemistry, University of Frankfurt, D-60438 Frankfurt, Germany absorption into and increased transport through small intestinal epithelial cells.

Keywords Absorption · Bioavailability · Caco- 2 cells · Curcumin micelles · Transepithelial transport · Tween 80

\section{Introduction}

Curcumin, the active constituent of Curcuma longa L. (family Zingiberaceae), has gained increasing interest because of its anti-inflammatory, anti-diabetic, anti-rheumatic, anti-oxidant, anti-cancer, wound healing, and hepatoprotective properties associated with good tolerability and safety up to very high doses of $12 \mathrm{~g}$ [1-4].

However, its low aqueous solubility, extensive intestinal and hepatic metabolism, as well as rapid urinary excretion limit its oral bioavailability to plasma concentrations in the nanomolar range, even when ingested at single oral doses of $10-12 \mathrm{~g}[5,6]$.

In order to overcome the low bioavailability of curcumin, a number of different strategies have been pursued to enhance its solubility, including the use of adjuvants to inhibit its metabolism [7], the application of crystalline curcumin in micronized form [8] and the incorporation of curcumin into phosphatidylcholine liposomes [9] or micelles [10, 11]. Nanoencapsulation of curcumin, using synthetic and naturalbased polymers has been mainly used for intravenous cancer therapy [12]. Only two studies reported improved bioavailability of PLGA-PEG encapsulated curcumin nanoparticles and a liposomal nanoparticle formulation in rats following oral administration $[13,14]$. Recently, curcumin-loaded selfassembled polymeric micelles were prepared using ditocopherol polyethylene glycol 2000 succinate (TPGS), HS15 (octadecanoic acid, 12-hydroxy-polymer with alpha- 
hydro-omega-hydroxypoly (oxy-1,2-ethanediyl)) and Pluronic F127 [15]. Another promising approach for the efficient delivery of poorly soluble substances is the preparation of nanoscaled micellar formulations based on Tween 80 , which enhance curcumin bioavailability up to 185 -fold in humans $[10,11]$. We therefore investigated the transepithelial transport and cellular accumulation of curcumin, demethoxycurcumin (DMC, curcumin II) and bis-demethoxycurcumin (BDMC, curcumin III) in their native form compared to a nanoscaled micellar formulation in a Caco- 2 cell model.

\section{Materials and Methods}

\section{Curcumin Formulations}

Native curcumin powder composed of $82 \%$ curcumin, $16 \%$ DMC and $2 \%$ BMDC used for the transport studies and for the preparation of the Tween 80 based micellar formulation was purchased from Jupiter Lys (Cochin, Kerala State, India). Sol-CUR consisting of $7 \%$ curcuminoid powder (equal to $6 \%$ curcumin) and $93 \%$ Tween-80 (Kolb, Hedingen, Switzerland) was produced by AQUANOVA AG (Darmstadt, Germany). A dose of $98 \mathrm{mg}$ total curcuminoids (80.36 mg curcumin, $15.68 \mathrm{mg}$ DMC and $1.96 \mathrm{mg}$ BDMC) was used for the transport experiments with native and micellar curcumin.

The sample solution of Sol-CUR used for the transport experiments was prepared by diluting $53 \mathrm{mg}$ of Sol-CUR with $10 \mathrm{~mL}$ modified FaSSIF (fasted state simulated intestinal fluid) transport buffer. The sample solution of CUR was prepared by diluting $32 \mathrm{mg}$ native curcumin powder with $10 \mathrm{~mL}$ modified FaSSIF transport buffer and further diluting an aliquot of $1 \mathrm{~mL}$ from that solution with $9 \mathrm{~mL}$ modified FaSSIF transport buffer. Both sample solutions yielded a curcumin concentration of $320 \mu \mathrm{g} / \mathrm{mL}(869 \mu \mathrm{mol} / \mathrm{L})$ corresponding to a dose of $80 \mathrm{mg}$ curcumin taken together with a glass of $250 \mathrm{~mL}$ water.

\section{Cell Culture Conditions}

Differentiated Caco-2 cells (passages 24-36; American Type Culture Collection (ATCC), Maryland, USA) were plated at a density of $6.5 \times 10^{4}$ cells per $\mathrm{cm}^{2}$ on 12 -well Transwell plates $\left(1.2 \mathrm{~cm}^{2}\right.$ polycarbonate membrane, $0.4 \mu \mathrm{m}$ pore size; Corning, NY, USA) and grown in Dulbecco's modified Eagle's medium (DMEM) containing $25 \mathrm{mM}$ glucose supplemented with $10 \%$ FCS (fetal calf serum), $1 \%$ NEAA (non essential amino acids) and $0.01 \%$ gentamycin (all from Biochrom AG, Berlin, Germany) in an atmosphere consisting of $90 \%$ relative humidity with $10 \% \mathrm{CO}_{2}$ at $37^{\circ} \mathrm{C}$.

Modified FaSSIF (fasted state simulated intestinal fluid), containing $3 \mathrm{mM}$ taurocholate sodium and $0.75 \mathrm{mM}$ phosphatidylcholine in dihydrogenphosphate sodium buffer $(28.66 \mathrm{mM})$, was prepared by diluting appropriate amounts of Phares SIF powder (Phares AG, Muttenz, Switzerland) with HBSS transport medium, composed of Hanks balanced salt solution (HBSS; Biochrom AG, Berlin, Germany) containing $20 \mathrm{mM}$ HEPES (4-(2-hydroxyethyl)-1piperazineethanesulfonic acid; Sigma-Aldrich, Steinheim, Germany) buffered at a $\mathrm{pH}$ of 7.4.

\section{Permeability Experiments}

Permeation was investigated in apical-to-basolateral direction (AB) at $37{ }^{\circ} \mathrm{C}$ ( $n=6$ each). During transport the plates were agitated on a shaker at $120 \mathrm{rpm}$ and kept at a constant temperature of $37^{\circ} \mathrm{C}$. Prior to each experiment, the Caco-2 monolayers were washed with HBSS. The transepithelial electrical resistance (TEER) was measured before and after the transport experiments. FITC-Dextran (mean MW 4400 g/mol, SigmaAldrich) was used to gauge the integrity of the monolayers and propranolol hydrochloride (Fagron, Barsbüttel, Germany) served as positive control for the functionality of every cell passage $(n=6)$. The validity of the Caco- 2 system in the present study was ensured by TEER values greater $250 \Omega \mathrm{cm}^{2}$ and an average $\mathrm{P}_{\text {app }}$ value for propranolol of $56.77 \times 10^{-6} \mathrm{~cm} / \mathrm{s}$ [16].

To estimate the mass flux, the receiver fluid $(1500 \mu \mathrm{L})$ was withdrawn after 15, 30, 45, 60, 90, 120 and $180 \mathrm{~min}$, and replaced by an equal volume of fresh buffer solution, respectively. The donor fluid $(500 \mu \mathrm{L})$ was removed at the end of the transport experiments to determine the remaining apical curcuminoid concentrations. Furthermore each transwell filter was vortexed in $0.5 \mathrm{~mL}$ methanol for $15 \mathrm{~min}$ at the end of the incubation time. The resulting cell lysate was subjected to HPLC analysis to determine the accumulated curcuminoids in the Caco-2 cells. In addition, the initial curcuminoid concentrations $\left(\mathrm{t}_{0}\right)$ were determined in the CUR and Sol-CUR sample solution prior to the transport experiments.

\section{Quantification of Curcumin}

Sample preparation and analysis of curcuminoids were previously reported in detail $[10,11]$. Briefly, one $\mathrm{mL}$ fluid was acidified with $10 \mu \mathrm{L} 6 \mathrm{M}$ hydrochloric acid and incubated with $100 \mu \mathrm{L}$ beta-glucuronidase type $\mathrm{H} 1$ from Helix pomatia $(1 \mathrm{mg} /$ $100 \mu \mathrm{L}$ in $0.1 \mathrm{M}$ sodium acetate buffer, Sigma-Aldrich Chemie $\mathrm{GmbH}$, Schnelldorf, Germany) for $45 \mathrm{~min}$ at $37^{\circ} \mathrm{C}$. After triplicate extraction with $95 \%$ ethyl acetate and $5 \%$ methanol $(v /$ v), supernatants were evaporated to dryness and resuspended in $150 \mu \mathrm{L}$ methanol, vortexed for $20 \mathrm{~s}$, stored in the dark for $10 \mathrm{~min}$, vortexed for $20 \mathrm{~s}$ and transferred to HPLC vials. Twenty $\mu \mathrm{L}$ of each sample was injected into the HPLC system.

Curcuminoids were quantified on a Jasco HPLC system (Jasco GmbH, Gross-Umstadt, Germany) with a fluorescence detector (excitation wavelength $426 \mathrm{~nm}$, emission wavelength $536 \mathrm{~nm}$ ) and separated on a Reprosil-Pur C18-AQ column $(150 \mathrm{~mm} \times 4 \mathrm{~mm}, 3 \mu \mathrm{m}$ particle size; Dr. Maisch $\mathrm{GmbH}$, 
Fig. 1 Experimental setting of the Caco-2 transport experiments

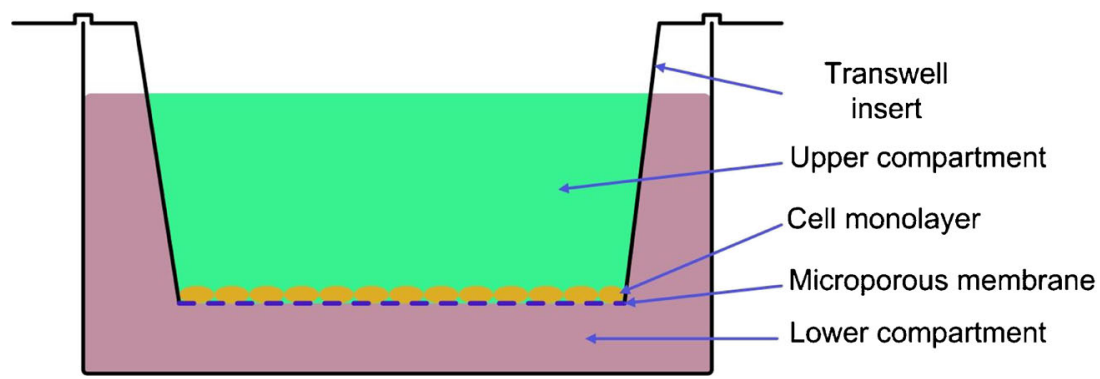

Ammerbuch, Germany) maintained at $40{ }^{\circ} \mathrm{C}$. The mobile phase consisted of $52 \%$ de-ionized water (adjusted to $\mathrm{pH} 3$ with perchloric acid), $34 \%$ acetonitrile and $14 \%$ methanol. Curcuminoids were quantified against external standard curves (curcumin, purity $\geq 97.2 \%$, CAS \# 458-37-7; DMC, purity $\geq 98.3 \%$, CAS \# 22,608-11-13; BDMC, purity $\geq 99.4 \%$, CAS \# 24,949-16-; Chromadex, Irvine, USA).

\section{Calculation of the Flux and Permeability Coefficient}

The flux was calculated over the linear range of the permeation curve for each well using the formula $(\mathrm{dc} / \mathrm{dt}) \times(1 / \mathrm{A})$, where $\mathrm{dc} / \mathrm{dt}$ indicates the slope of the permeation and $\mathrm{A}$ the surface area of the monolayer.

The permeability coefficient $\mathrm{P}_{\text {app }}(\mathrm{cm} / \mathrm{s})$ was calculated as follows: $(\mathrm{dC} / \mathrm{dt}) \times(\mathrm{Vr} / \mathrm{AC} 0)$, where $\mathrm{dc} / \mathrm{dt}$ is the flux rate $(\mu \mathrm{g} /$ $(\mathrm{mL} \times \mathrm{s}))$ through the monolayer, $\mathrm{Vr}$ is the volume of the receiver chamber $(\mathrm{mL}), \mathrm{A}$ is the surface area of the cell monolayer, and $\mathrm{C}_{0}$ is the initial concentration of the donor fluid $(\mu \mathrm{g} / \mathrm{mL})$.

\section{Results and Discussion}

The Caco- 2 cell system, being recommended by the US Food and Drug Administration (FDA), is an established and widely accepted in vitro tool for predicting the intestinal absorption of organic substances (Fig. 1) [17-19]. In the present study, curcumin was dissolved in modified FASSIF to simulate the gastrointestinal content at the site of absorption in the fasted state. No additional solubility enhancing surfactants were used as modified FASSIF already contains taurocholate sodium and lecithin as solubilizing enhancers.

At the end of the transport experiments, Sol-CUR afforded a nearly 9.5 fold higher concentration of curcumin on the basolateral side (transepithelial transport) compared to CUR, while the amount of curcumin accumulated in the Caco- 2 cells was much lower with Sol-CUR than CUR (Fig. 2), suggesting that the nanoscaled micellar formulation does not only increase the cellular uptake, but also the basolateral secretion of curcumin.

The higher amount of Sol-CUR on the basolateral site results from a higher transport rate compared to CUR and a higher flux of $39.23 \mu \mathrm{g} \mathrm{min}{ }^{-1} \mathrm{~cm}^{-2}$ for Sol-CUR compared to $4.98 \mu \mathrm{g} \mathrm{min}^{-1} \mathrm{~cm}^{-2}$ for CUR (Fig. 3). $\mathrm{P}_{\text {app }}$ values for SolCUR and CUR were $2.11 \times 10^{-6} \mathrm{~cm} / \mathrm{s}$ and $0.56 \times 10^{-6} \mathrm{~cm} / \mathrm{s}$, respectively. Also, the permeability of Caco- 2 cells for BDMC and $\mathrm{DMC}$ was increased as demonstrated by the higher $\mathrm{P}_{\mathrm{app}}$ values obtained for BDMC and DMC in the nanoscaled micellar formulation compared to native curcumin (Table 1).

Compared to the $\mathrm{P}_{\mathrm{app}}$ values for native curcumin of $0.05 \times 10^{-6} \mathrm{~cm} / \mathrm{s}$ and $0.07 \times 10^{-6} \mathrm{~cm} / \mathrm{s}$ reported in the
Fig. 2 Curcumin concentrations $(n=6$; mean $\pm \mathrm{SD})$ in the apical and basolateral compartments and in the cell pellets of Caco-2 cells incubated for $180 \mathrm{~min}$ with identical doses of native (CUR) and nanoscaled micellar curcumin (Sol-CUR)

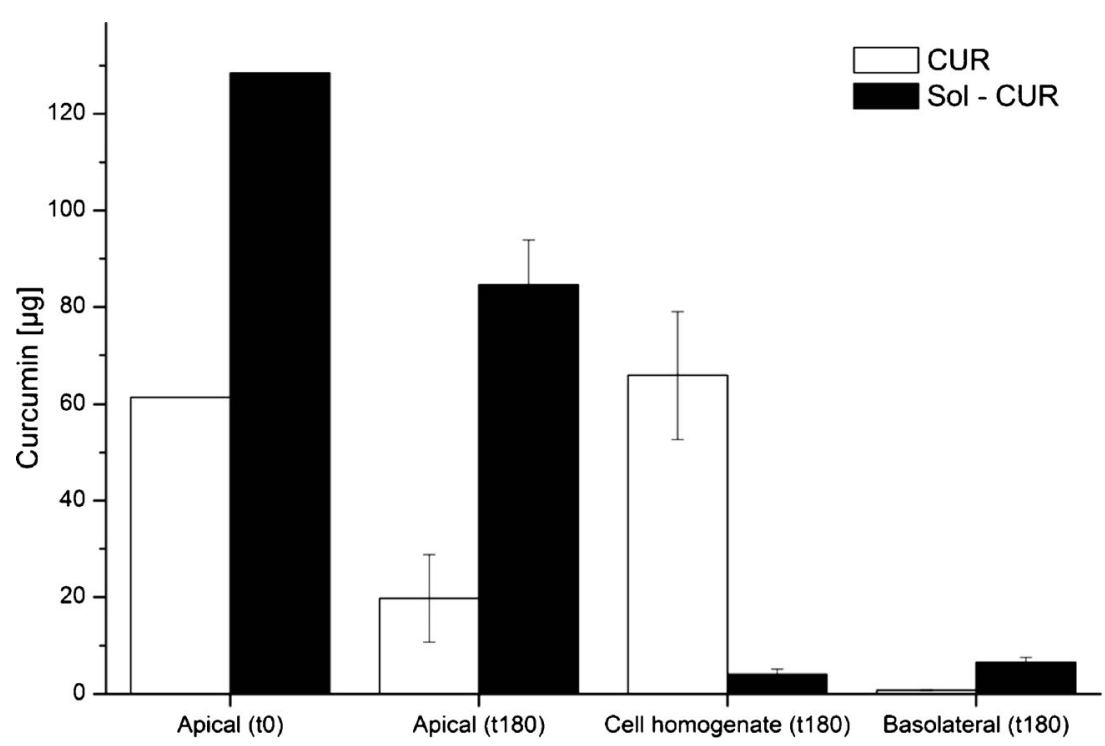


literature by Wang et al. and Dempe et al., respectively, the $P_{\text {app }}$ value determined in our study was higher $[15,20]$. These differences may be attributed to differences in the experimental conditions. Transport experiments in both studies were performed with HBSS on the apical side, which is supplemented with glucose $(25 \mathrm{mM})$ and buffered with $10 \mathrm{mM}$ HEPES at a $\mathrm{pH}$ of 7.4. Although frequently used in Caco-2 transport experiments, this buffer does not reflect the physiological conditions in vivo, since the absence of bile salts may limit the solubility of highly lipophilic substances and may thus affect the outcome of the Caco-2 transport experiments [21]. Moreover this buffer promotes adsorption and/or nonspecific binding of highly lipophilic substances to device surfaces, especially in the absence of sink conditions [22]. In order to overcome these drawbacks, the Caco-2 model used in this study was adapted to more closely mimic physiological conditions. This implied the provision of sink conditions as in vivo, i.e., a sufficiently diluted dissolution system in the receiver compartment that does not impede the dissolution of curcumin by approaching saturation. The addition of $4 \%$ BSA provided the necessary driving force, similar to in vivo, where the drug absorbed across the intestinal epithelium is immediately carried away by the portal blood containing about 4\% albumin [21]. Further adaptation to in vivo conditions was achieved by using modified FASSIF instead of pure HBSS in the apical compartment. The taurocholate sodium and lecithin included in modified FASSIF simulate the colloidal mixture of bile salts and phosphatidylcholine in the intestine, which are responsible for enhanced solubilisation effects in vivo. Thus, the enhanced initial solubility of curcumin in the apical compartment in combination with the provided sink conditions, led to a higher $\mathrm{P}_{\text {app }}$ value for native curcumin than that published before $[15,20]$. A higher $\mathrm{P}_{\text {app }}$ value for native curcumin of $2.9 \times 10^{-6} \mathrm{~cm} / \mathrm{s}$ was reported only in one study, which may be explained by the much higher initial concentration of curcumin of $170 \mu \mathrm{mol} / \mathrm{L}$ used, a transition time of only $120 \mathrm{~min}$ and a pH of 6.5 in the apical compartment at which curcumin is more stable [23].

The $\mathrm{P}_{\text {app }}$ value of $2.11 \times 10^{-6} \mathrm{~cm} / \mathrm{s}$ for Sol-CUR observed in the present study, indicating a 3.8 -fold improvement in the permeability of micellar curcumin over the native form, suggests that the enhanced oral bioavailability of micellar curcumin formulations reported in two human pharmacokinetic studies, resulting in an up to 185 -fold increase in the AUC compared to native curcumin $[10,11]$, is likely a result of enhanced absorption into and increased transport through small intestinal epithelial cells. The present study is the first to provide insights into the processes underlying the observed enhanced bioavailability of nanoscaled micellar curcumin formulations in humans, underlining thus the potential of the Caco-2 cell model to serve as an excellent predictive tool for the oral absorption of not only native compounds, but also more complex drug-excipient formulations.

Also curcumin-loaded self-assembled polymeric micelles made from TPGS2 K, HS15, and Pluronic F127 facilitated a 3.5- fold increase in permeability compared to native curcumin [15]. In fact, both TPGS2 K and Tween 80 are non-ionic surfactants improving the bioavailability of poorly soluble drugs by micellar solubilisation [23]. However, Tween 80-based micellar formulations are characterized by a better surfactant to curcumin ratio. While the curcumin-loaded selfassembled polymeric micelles consisted of curcumin, TPGS2 K, HS15 and Pluronic F127 in a ratio of $1: 10: 10: 2$ (ca. $4 \%$ curcuminoids corresponding to $3 \%$ curcumin) [15], the Tween 80 micellar formulation consisted of $7 \%$ curcuminoids (corresponding to $6 \%$ curcumin) and 93\% Tween- 80 .
Fig. 3 Curcumin flux ( $\mu \mathrm{g} \mathrm{min}^{-1} \mathrm{~cm}^{-2}$ ) of native (CUR) and nanoscaled micellar curcumin. Sol-CUR across Caco2 monolayers $(n=6$; mean \pm S.D.)

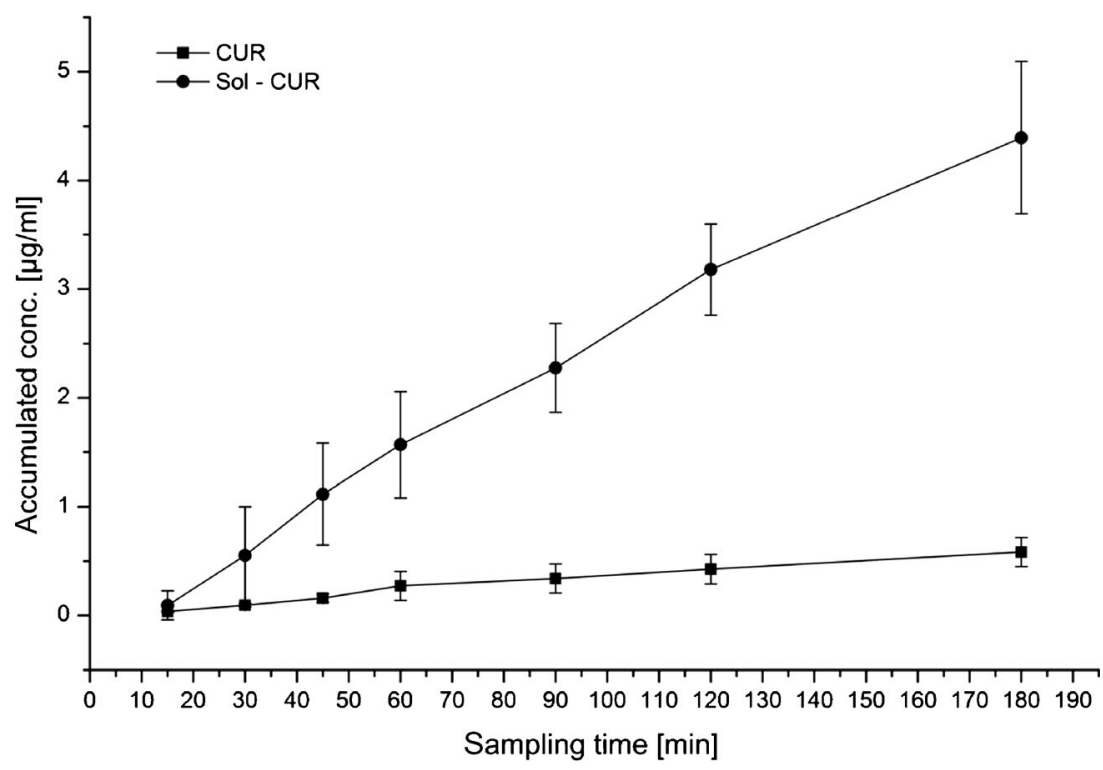


Table $1 \mathrm{P}_{\text {app }}$ values (expressed as $10^{-6} \mathrm{~cm} / \mathrm{s}$ ) calculated for curcumin, $\mathrm{DMC}$ and BDMC in native curcumin (CUR) and nanoscaled micellar formulation of curcumin (Sol-CUR)

\begin{tabular}{lll}
\hline Analyte & CUR & Sol-CUR \\
\hline Curcumin & $0.56 \pm 0.14$ & $2.11 \pm 0.34$ \\
DMC & $1.32 \pm 0.11$ & $3.61 \pm 0.59$ \\
BDMC & $1.91 \pm 0.25$ & $6.63 \pm 0.67$ \\
\hline
\end{tabular}

Consequently a lower amount of surfactants is required in the nanoscaled Tween 80 micellar formulation than in case of the curcumin-loaded self-assembled polymeric micelles. This is of special benefit as the overall amount of surfactants administered orally may thus be reduced.

At the same time the increase in bioavailability achieved with the nanoscaled Tween 80 micellar formulation is much higher than with the curcumin-loaded-self-assembled polymeric micelles. Thus, an up to 185 -fold increase in the AUC of curcumin was observed in humans with the nanoscaled micellar formulation, whereas the curcumin-loaded-selfassembled polymeric micelles achieved only a 2.87 -fold increase in the AUC of curcumin compared to the native compound $[10,11,15]$. Moreover, Tween 80 is much cheaper than TPGS, rendering it a cost-effective option to enhance the delivery of poorly soluble compounds to target tissues.

\section{Conclusion}

The present study provides insight into the processes underlying the observed 185-fold enhanced bioavailability of nanoscaled micellar curcumin formulation in two previously conducted human pharmakokinetic studies. The increase in the apparent permeability coefficient observed for micellar over native curcumin in the Caco- 2 in vitro system results from a higher transport rate and a higher flux for micellar curcumin through the intestinal barrier. Also the amount of curcumin accumulated in the Caco-2 cells was much lower in case of nanoscaled micellar curcumin, suggesting that the micellation of curcumin does not only increase the cellular uptake but also the basolateral secretion of curcumin.

\section{Compliance with Ethical Standards}

Conflict of Interest The study was financially supported by AQUANOVA AG (Darmstadt, Germany) and the German Federal Ministry of Education and Research (grant \# 01EA1334A). Jan Frank is a consultant to AQUANOVA AG, the company manufacturing micellar curcumin. Dariush Behnam is the founder and CEO of AQUANOVA AG, the company producing and selling the curcuminoid micelles for profit. Mr. Behnam was not involved in data analysis and interpretation, which were exclusively in the hands of the academic authors. All other authors have stated not to have any known conflict of interest.
Open Access This article is distributed under the terms of the Creative Commons Attribution 4.0 International License (http:// creativecommons.org/licenses/by/4.0/), which permits unrestricted use, distribution, and reproduction in any medium, provided you give appropriate credit to the original author(s) and the source, provide a link to the Creative Commons license, and indicate if changes were made.

\section{References}

1. Shehzad A, Wahid F, Lee YS (2010) Curcumin in cancer chemoprevention: molecular targets, pharmacokinetics, bioavailability, and clinical trials. Arch Pharm Chem Life Sci 9:489-499

2. Siviero A, Gallo E, Maggini V, Gori L, Mugelli A, Firenzuoli F, Vannacci A (2015) Curcumin, a golden spice with a low bioavailability. J Herb Med 5:57-70

3. Aggarwal BB, Sundaram C, Malani N, Ichikawa H (2007) Curcumin: the Indian solid gold. Adv Exp Med Biol 595:1-75

4. Tundis R, Loizzo MR, Bonesi M, Menichini F (2015) Potential role of natural compounds against skin aging. Curr Med Chem 22: $1515-1538$

5. Vareed SK, Kakarala M, Ruffin MT, Crowell JA, Normolle DP, Djuric Z, Brenner DE (2008) Pharmacokinetics of curcumin conjugate metabolites in healthy human subjects. Cancer Epidemiol Biomarkers 17:11-17

6. Sun M, Su X, Ding B, He X, Liu X, Yu A, Lou H, Zhai G (2012) Advances in namotechnology-based delivery systems for curcumin. Nanomedicine 7:1085-1100

7. Shoba G, Joy D, Joseph T, Majeed M, Rajendran R, Srinivas PS (1998) Influence of piperine on the pharmacokinetics of curcumin in animals and human volunteers. Planta Med 65:353-356

8. Sasaki H, Sunagawa Y, Takahashi K, Imaizumi A, Fukuda H, Hashimoto T, Wada H, Katanasaka Y, Kakeya H, Fujita M, Hasegawa K, Morimoto T (2011) Innovative preparation of curcumin for improved oral bioavailability. Biol Pharm Bull 34: 660-665

9. Cuomo J, Appendino G, Dern AS, Schneider E, McKinnon TP, Brown MJ, Togni S, Dixon BM (2011) Comparative absorption of a standardized curcuminoid mixture and its lecithin formulation. J Nat Prod 74:664-669

10. Kocher A, Schiborr C, Behnam D, Frank J (2015) The oral bioavailybility of curcuminoids in healthy humans is markedly enhanced by micellar solubilisation but not further imporved by simultaneous ingestion of sesamin, ferulic acid, naringenin and xanthohumol. J Funct Foods 14:183-191

11. Schiborr C, Kocher A, Behnam D, Jandasek J, Toelstede S, Frank J (2014) The oral bioavailability of curcumin from micronized powder and liquid micelles is significantly increased in healthy humans and differs between sexes. Mol Nutr Food Res 58:516-527

12. Lee WH, Loo CY, Young PM, Traini D, Mason RS, Rohanizadeh R (2014) Recent advances in curcumin nanoformulation for cancer therapy. Expert Opin Drug Deliv 11:1183-1201

13. Khalil NM, do Nascimento TC, Casa DM, Dalmolin LF, de Mattos AC, Hoss I, Romano MA, Mainardes RM (2013) Pharmacokinetics of curcumin-loaded PLGA and PLGA-PEG blend nanoparticles after oral administration in rats. Colloids Surf B 101:353-360

14. Takahashi M, Uechi S, Takara K, Asikin Y, Wada K (2009) Evaluation of an oral carrier system in rats: bioavailability and antioxidant properties of liposome-encapsulated curcumin. J Agric Food Chem 57:9141-9146

15. Wang J, Ma W, Tu P (2015) The mechanism of self-assembled mixed micelles in improving curcumin oral absorption: in vitro and in vivo. Colloids Surf B Biointerfaces 133:108-119 
16. Yee $\mathrm{S}$ (1997) In vitro permeability across Caco-2 cells (colonic) can predict in vivo (small intestinal) absorption in man - fact or myth. Pharm Res 14:763-766

17. Artursson P (1990) Epithelial transport of drugs in cell culture. I: a model for studying the passive diffusion of drugs over intestinal absorptive (Caco-2) cells. J Pharm Sci 79:476-482

18. Artursson P, Palm K, Luthman K (2001) Caco-2 monolayers in experimental and theoretical predictions of drug transport. Adv Drug Deliv Rev 46:27-43

19. Center for Drug Evaluation and Research, Food and Drug Administration, Rockville, MD, Guidance for Industry (2000) Waiver of in vivo bioavailability and bioequivalence studies for immediate-release solid oral dosage forms based on a biopharmaceutics classification system
20. Dempe JS, Scheerle RK, Pfeiffer E, Metzker M (2013) Metabolism and permeability of curcumin in cultured Caco-2 cells. Mol Nutr Food Res 57:1543-1549

21. Krishna G, K-j C, Lin C-c, Nomeir AA (2001) Permeability of lipophilic compounds in drug discovery using in vitro human absorptoin model, Caco-2. Int J Pharm 222:77-89

22. Ingels FM, Augustijns PF (2003) Biological, pharmaceutical, and analytical considerations with respect to the transport media used in the absorption screening system, Caco-2. J Pharm Sci 92:1545-1558

23. Wahlang B, Pawar YB, Bansal AK (2011) Identification of permeability-hurdles in oral delivery of curcumin using the Caco2 model. Eur J Pharm Biopharm 77:275-282 